\title{
Solutions for a variational inclusion problem with applications to multiple sets split feasibility problems
}

\author{
Lai-Jiu Lin ${ }^{1 *}$, Yew-Dann Chen ${ }^{1,2}$ and Chih-Sheng Chuang ${ }^{3}$
}

\author{
"Correspondence: \\ maljlin@cc.ncue.edu.tw \\ 1 Department of Mathematics, \\ National Changhua University of \\ Education, Changhua, 50058, \\ Taiwan \\ Full list of author information is \\ available at the end of the article
}

\begin{abstract}
In this paper, we first study the set of common solutions for two variational inclusion problems in a real Hilbert space and establish a strong convergence theorem of this problem. As applications, we study unique minimum norm solutions of the following problems: multiple sets split feasibility problems, system of convex constrained linear inverse problems, convex constrained linear inverse problems, split feasibility problems, convex feasibility problems. We establish iteration processes of these problems and show the strong convergence theorems of these iteration processes. MSC: 47J20; 47J25; 47H05; 47H09

Keywords: firmly nonexpansive mapping; proximal-contraction; multiple sets split feasibility problem; maximum monotone; convex feasibility problem
\end{abstract}

\section{Introduction}

Let $C_{1}, C_{2}, \ldots, C_{m}$ be nonempty closed convex subsets of a real Hilbert space $\mathcal{H}$. The wellknown convex feasibility problem (CFP) is to find $x^{*} \in \mathcal{H}$ such that

$$
x^{*} \in C_{1} \cap C_{2} \cap \cdots \cap C_{m} .
$$

The convex feasibility problem has received a lot of attention due to its diverse applications in mathematics, approximation theory, communications, geophysics, control theory, biomedical engineering. One can refer to $[1,2]$.

If $C_{1}$ and $C_{2}$ are closed vector spaces of a real Hilbert space $\mathcal{H}$, in 1933, von Neumann showed that any sequence $\left\{x_{n}\right\}$ is generated from the method of alternating projections: $x_{0} \in \mathcal{H}, x_{1}=P_{C_{1}} x_{0}, x_{2}=P_{C_{2}} x_{1}, x_{3}=P_{C_{1}} x_{2}, \ldots$ Then $\left\{x_{n}\right\}$ converges strongly to some $\bar{x} \in$ $C_{1} \cap C_{2}$. If $C_{1}$ and $C_{2}$ are nonempty closed convex subsets of $\mathcal{H}$, Bregman [3] showed that the sequence $\left\{x_{n}\right\}$ generated from the method of alternating projection converges weakly to a point in $C_{1} \cap C_{2}$. Hundal [4] showed that the strong convergence fails if $C_{1}$ and $C_{2}$ are nonempty closed convex subsets of $\mathcal{H}$. Recently, Boikanyo et al. [5] proposed the following process:

$$
x_{2 n+1}=J_{\beta_{n}}^{G_{1}}\left(\alpha_{n} u+\left(1-\alpha_{n}\right) x_{2 n}+e_{n}\right), \quad n=0,1, \ldots
$$

\section{Springer}

○2013 Lin et al.: licensee Springer. This is an Open Access article distributed under the terms of the Creative Commons Attribution License (http://creativecommons.org/licenses/by/2.0), which permits unrestricted use, distribution, and reproduction in any medium, provided the original work is properly cited. 
and

$$
x_{2 n}=J_{\rho_{n}}^{G_{2}}\left(\lambda_{n} u+\left(1-\lambda_{n}\right) x_{2 n-1}+e_{n}^{\prime}\right), \quad n=0,1, \ldots
$$

where $u, x_{0} \in \mathcal{H}$ are given arbitrarily, $G_{1}$ and $G_{2}$ are two set-valued maximal monotone operators with $J_{\beta}^{G_{1}}=\left(I+\beta G_{1}\right)^{-1}$, and $\left\{\alpha_{n}\right\},\left\{\lambda_{n}\right\},\left\{\beta_{n}\right\},\left\{\rho_{n}\right\},\left\{e_{n}\right\},\left\{e_{n}^{\prime}\right\}$ are sequences. Boikanyo et al. [5] proved that the sequence $\left\{x_{n}\right\}$ converges strongly to a point $\bar{x} \in G_{1}^{-1}(0) \cap G_{2}{ }^{-1}(0)$ under suitable conditions.

The split feasibility problem (SFP) is to find a point

$$
x^{*} \in C \text { such that } A x^{*} \in Q \text {, }
$$

where $C, Q$ are nonempty closed convex subsets of real Hilbert spaces $\mathcal{H}_{1}, \mathcal{H}_{2}$, respectively. $A: \mathcal{H}_{1} \rightarrow \mathcal{H}_{2}$ is a bounded linear operator. The split feasibility problem (SFP) in finite dimensional real Hilbert spaces was first introduced by Censor and Elfving [6] for modeling inverse problems which arise from medical image reconstruction. Since then, the split feasibility problem (SFP) has received much attention due to its applications in signal processing, image reconstruction, approximation theory, control theory, biomedical engineering, communications, and geophysics. For examples, one can refer to [1, 2, 6-17] and related literature. A special case of problem (SFP) is the convexly constrained linear inverse problem in a finite dimensional real Hilbert space [18]:

(CLIP) Find $\bar{x} \in C$ such that $A \bar{x}=b$,

where $C$ is a nonempty closed convex subset of a real Hilbert space $\mathcal{H}_{1}$ and $b$ is a given element of a real Hilbert space $\mathcal{H}_{2}$, which has extensively been investigated by using the Landweber iterative method [19]:

$$
x_{n+1}:=x_{n}+\gamma A^{T}\left(b-A x_{n}\right), \quad n \in \mathbb{N} .
$$

Let $C_{1}, C_{2}, \ldots, C_{m}$ be nonempty closed convex subsets of $\mathcal{H}_{1}$, let $Q_{1}, Q_{2}, \ldots, Q_{m}$ be nonempty closed convex subsets of $\mathcal{H}_{2}$, and let $A_{1}, A_{2}, \ldots, A_{m}: \mathcal{H}_{1} \rightarrow \mathcal{H}_{2}$ be bounded linear operators. The well-known multiple sets split feasibility problem (MSSFP) is to find $x^{*} \in \mathcal{H}_{1}$ such that

$$
x^{*} \in C_{i} \text { such that } A_{i} x^{*} \in Q_{i} \text { for all } i=1,2, \ldots, m \text {. }
$$

The multiple sets split feasibility problem (MSSFP) contains convex feasibility problem (CFP) and split feasibility problem (SFP) as special cases [6, 10, 13]. Indeed, Censor et al. [11] first studied this type of problem. Xu [16] and Lopez et al. [13] also studied this type of problem. In 2011, Boikanyo and Moroşanu [20] gave the following algorithm:

$$
\begin{cases}v_{2 n+1}:=a_{n} u+b_{n} v_{2 n}+c_{n} J_{\alpha_{n}}^{G_{1}} v_{2 n}, & n \in \mathbb{N} \cup\{0\} \\ v_{2 n}:=f_{n} u+g_{n} v_{2 n-1}+h_{n} J_{\beta_{n}}^{G_{2}} v_{2 n-1}, & n \in \mathbb{N}\end{cases}
$$

where $u$ is given in $\mathcal{H}$, and $G_{1}, G_{2}$ are two set-valued maximal monotone mappings on $\mathcal{H},\left\{a_{n}\right\},\left\{b_{n}\right\},\left\{c_{n}\right\},\left\{f_{n}\right\},\left\{g_{n}\right\}$ and $\left\{h_{n}\right\}$ are sequences in [0,1]. Boikanyo and Moroşanu [20] 
proved that $\left\{v_{n}\right\}$ in (1.3) converges strongly to some $\bar{x} \in G_{1}^{-1}(0) \cap G_{2}{ }^{-1}(0)$ under suitable conditions.

Motivated by the above works, we consider the following algorithm:

$$
\left\{\begin{array}{l}
v_{0} \in \mathcal{H} \text { is chosen arbitrarily, } \\
v_{2 n+1}:=a_{n} u+b_{n} v_{2 n}+c_{n} J_{\delta_{n}}^{G_{1}}\left(I-\delta_{n} B_{1}\right) v_{2 n}, \quad n \in \mathbb{N} \cup\{0\}, \\
v_{2 n}:=f_{n} u+g_{n} v_{2 n-1}+h_{n} J_{\gamma_{n}}^{G_{2}}\left(I-\gamma_{n} B_{2}\right) v_{2 n-1}, \quad n \in \mathbb{N},
\end{array}\right.
$$

where $G_{1}, G_{2}$ are two set-valued maximal monotone mappings on a real Hilbert space $\mathcal{H}_{1}, B_{1}, B_{2}: C \rightarrow \mathcal{H}_{1}$ are two mappings, $\left\{a_{n}\right\},\left\{b_{n}\right\},\left\{c_{n}\right\},\left\{f_{n}\right\},\left\{g_{n}\right\}$, and $\left\{h_{n}\right\}$ are sequences in $[0,1]$. We show that the sequence $\left\{v_{n}\right\}$ generated by (1.4) converges strongly to some $\bar{x} \in\left(B_{1}+G_{1}\right)^{-1}(0) \cap\left(B_{2}+G_{2}\right)^{-1}(0)$ under suitable conditions.

Algorithm (1.4) contains as special cases the inexact proximal point algorithm (e.g., [21, $22]$ ) and the generalized contraction proximal point algorithm (e.g., [23]). Our conclusion extends and unifies Boikanyo and Moroşanu's result in [20], Wang and Cui's result in [24] becomes a special case. For details, one can see Section 3.

In this paper, we first study the set of common solutions for two variational inclusion problems in a Hilbert space and establish a strong convergence theorem of this problem. As applications, we study unique minimum norm solutions of the following problems: multiple sets split feasibility problems, system of convex constrained linear inverse problems, convex constrained linear inverse problems, split feasibility problems, convex feasibility problems. We establish iteration processes of these problems and show strong convergence theorems of these iteration processes.

\section{Preliminaries}

Throughout this paper, let $\mathcal{H}, \mathcal{H}_{1}, \mathcal{H}_{2}, \mathcal{H}_{3}$ denote real Hilbert spaces with the inner product $\langle\cdot, \cdot\rangle$ and the norm $\|\cdot\|$; let $\mathbb{N}$ be the set of all natural numbers and $\mathbb{R}^{+}$be the set of all positive real numbers. A set-valued mapping $A$ with domain $\mathcal{D}(A)$ on $\mathcal{H}$ is called monotone if $\langle u-v, x-y\rangle \geq 0$ for any $u \in A x, v \in A y$ and for all $x, y \in \mathcal{D}(A)$. A monotone operator $A$ is called maximal monotone if its graph $\{(x, y): x \in \mathcal{D}(A), y \in A x\}$ is not properly contained in the graph of any other monotone mapping. The set of all zero points of $A$ is denoted by $A^{-1}(0)$, i.e., $A^{-1}(0)=\{x \in \mathcal{H}: 0 \in A x\}$. In what follows, we denote the strong convergence and the weak convergence of $\left\{x_{n}\right\}$ to $x \in \mathcal{H}$ by $x_{n} \rightarrow x$ and $x_{n} \rightarrow x$, respectively. In order to facilitate our discussion, in the next section, we recall some facts. The following equality is easy to check:

$$
\begin{aligned}
& \|\alpha x+\beta y+\gamma z\|^{2} \\
& \quad=\alpha\|x\|^{2}+\beta\|y\|^{2}+\gamma\|z\|^{2}-\alpha \beta\|x-y\|^{2}-\alpha \gamma\|x-z\|^{2}-\beta \gamma\|y-z\|^{2}
\end{aligned}
$$

for each $x, y, z \in \mathcal{H}$ and $\alpha, \beta, \gamma \in[0,1]$ with $\alpha+\beta+\gamma=1$. Besides, we also have

$$
\|x+y\|^{2} \leq\|x\|^{2}+2\langle y, x+y\rangle
$$

for each $x, y \in \mathcal{H}$. Let $C$ be a nonempty closed convex subset of $\mathcal{H}$, and a mapping $T: C \rightarrow \mathcal{H}$. We denote the set of all fixed points of $T$ by $\operatorname{Fix}(T)$. A mapping $T: C \rightarrow \mathcal{H}$ is said to be nonexpansive if $\|T x-T y\| \leq\|x-y\|$ for every $x, y \in C$. A mapping $T: C \rightarrow \mathcal{H}$ 
is said to be quasi-nonexpansive if $\operatorname{Fix}(T) \neq \emptyset$ and $\|T x-y\| \leq\|x-y\|$ for all $x \in C$ and $y \in \operatorname{Fix}(T)$. A mapping $T: C \rightarrow \mathcal{H}$ is said to be firmly nonexpansive if

$$
\|T x-T y\|^{2} \leq\|x-y\|^{2}-\|(I-T) x-(I-T) y\|^{2}
$$

for every $x, y \in C$. Besides, it is easy to see that $\operatorname{Fix}(T)$ is a closed convex subset of $C$ if $T$ : $C \rightarrow \mathcal{H}$ is a quasi-nonexpansive mapping. A mapping $T: C \rightarrow \mathcal{H}$ is said to be $\alpha$-inversestrongly monotone $(\alpha$-ism) if

$$
\langle x-y, T x-T y\rangle \geq \alpha\|T x-T y\|^{2}
$$

for all $x, y \in \mathcal{H}$ and $\alpha>0$.

The following lemmas are needed in this paper.

Lemma 2.1 [25] Let $A: \mathcal{H}_{1} \rightarrow \mathcal{H}_{2}$ be a bounded linear operator, and let $A^{*}$ be the adjoint of $A$. Suppose that $C$ is a nonempty closed convex subset of $\mathcal{H}_{2}$, and that $G: C \rightarrow \mathcal{H}_{2}$ is a firmly nonexpansive mapping. Then $A^{*}(I-G) A$ is $\frac{1}{\|A\|^{2}}$-ism, that is,

$$
\frac{1}{\|A\|^{2}}\left\|A^{*}(I-G) A x-A^{*}(I-G) A y\right\|^{2} \leq\left\langle x-y, A^{*}(I-G) A x-A^{*}(I-G) A y\right\rangle
$$

for all $x, y \in \mathcal{H}_{1}$.

Lemma 2.2 Let $C$ be a nonempty closed convex subset of $\mathcal{H}$, and let $G: C \rightarrow \mathcal{H}$ be a firmly nonexpansive mapping. Suppose that $\operatorname{Fix}(G)$ is nonempty. Then $\langle x-G x, G x-w\rangle \geq 0$ for each $x \in \mathcal{H}$ and each $w \in \operatorname{Fix}(G)$.

Let $C$ be a nonempty closed convex subset of $\mathcal{H}$. Then, for each $x \in \mathcal{H}$, there is a unique element $\bar{x} \in C$ such that $\|x-\bar{x}\|=\min _{y \in C}\|x-y\|$. Here, we set $P_{C} x=\bar{x}$ and $P_{C}$ is said to be the metric projection from $\mathcal{H}$ onto $C$.

Lemma 2.3 [26] Let $C$ be a nonempty closed convex subset of $\mathcal{H}$, and let $P_{C}$ be the metric projection from $\mathcal{H}$ onto $C$. Then $\left\langle x-P_{C} x, P_{C} x-y\right\rangle \geq 0$ for each $x \in \mathcal{H}$ and each $y \in C$.

For a set-valued maximal monotone operator $G$ on $\mathcal{H}$ and $r>0$, we may define an operator $J_{r}^{G}: \mathcal{H} \rightarrow \mathcal{H}$ with $J_{r}^{G}=(I+r G)^{-1}$ which is called the resolvent mapping of $G$ for $r$.

Lemma 2.4 $[26,27]$ Let $G: \mathcal{H} \rightarrow 2^{\mathcal{H}}$ be a set-valued maximal monotone mapping. Then we have that

(i) for each $\alpha>0, J_{\alpha}^{G}$ is a single-valued and firmly nonexpansive mapping.

(ii) $\mathcal{D}\left(J_{\alpha}^{G}\right)=\mathcal{H}$ and $\operatorname{Fix}\left(J_{\alpha}^{G}\right)=G^{-1}(0)$.

(iii) $\left\|x-J_{\alpha}^{G} x\right\| \leq 2\left\|x-J_{\beta}^{G} x\right\|$ for each $x \in \mathcal{H}$ and all $\alpha, \beta \in(0, \infty)$ with $0<\alpha \leq \beta$.

Lemma 2.5 [26] Let $G$ be a set-valued maximal monotone operator on $\mathcal{H}$. For $a>0$, we define the resolvent $J_{a}^{G}=(I+a G)^{-1}$. Then the following holds:

$$
\left\|J_{\alpha}^{G} x-J_{\beta}^{G} x\right\|^{2} \leq \frac{\alpha-\beta}{\alpha}\left\langle J_{\alpha}^{G} x-J_{\beta}^{G} x, J_{\alpha}^{G} x-x\right\rangle
$$


for all $\alpha, \beta>0$ and $x \in \mathcal{H}$. In fact,

$$
\left\|J_{\alpha}^{G} x-J_{\beta}^{G} x\right\| \leq \frac{|\alpha-\beta|}{\alpha}\left\|J_{\alpha}^{G} x-x\right\|
$$

for all $\alpha, \beta>0$ and $x \in \mathcal{H}$.

Lemma 2.6 [28] Let $\left\{S_{n}\right\}$ be a sequence of real numbers that does not decrease at infinity, in the sense that there exists a subsequence $\left\{S_{n_{i}}\right\}_{i \geq 0}$ of $\left\{S_{n}\right\}$ such that $S_{n_{i}}<S_{n_{i}+1}$ for all $i \in \mathbb{N}$. Consider the sequence $\{\tau(n)\}_{n \geq n_{0}}$ defined by $\tau(n)=\max \left\{n_{0} \leq k \leq n: \tau_{k}<\tau_{k+1}\right\}$ for some sufficiently large number $n_{0} \in \mathbb{N}$. Then $\{\tau(n)\}_{n \geq n_{0}}$ is a nondecreasing sequence with $\tau(n) \rightarrow \infty$ as $n \rightarrow \infty$, and for all $n \geq n_{0}$,

$$
S_{\tau(n)} \leq S_{\tau(n)+1} \quad \text { and } \quad S_{n} \leq S_{\tau(n)+1} .
$$

In fact, $\max \left\{S_{\tau(n)}, S_{n}\right\} \leq S_{\tau(n)+1}$.

Lemma 2.7 [5] Let $\left\{a_{n}\right\}$ and $\left\{b_{n}\right\}$ be two sequences in $[0,1]$. Let $\left\{e_{n}\right\}$ be a sequence of nonnegative real numbers. Let $\left\{t_{n}\right\}$ and $\left\{k_{n}\right\}$ be two sequences of real numbers. Let $\left\{S_{n}\right\}_{n \in \mathbb{N}}$ be a sequence of nonnegative real numbers with

$$
S_{n+1} \leq\left(1-a_{n}\right)\left(1-b_{n}\right) S_{n}+a_{n} t_{n}+b_{n} k_{n}+e_{n}
$$

for each $n \in \mathbb{N}$. Assume that:

$$
\sum_{n=1}^{\infty} a_{n}=\infty, \quad \limsup _{n \rightarrow \infty} t_{n} \leq 0, \quad \limsup _{n \rightarrow \infty} k_{n} \leq 0, \quad \text { and } \quad \sum_{n=1}^{\infty} e_{n}=\infty .
$$

Then $\lim _{n \rightarrow \infty} S_{n}=0$.

A mapping $T: \mathcal{H} \rightarrow \mathcal{H}$ is said to be averaged if $T=(1-\alpha) I+\alpha S$, where $S: \mathcal{H} \rightarrow \mathcal{H}$ is a nonexpansive mapping and $\alpha \in(0,1)$.

Lemma 2.8 [29] Let $C$ be a nonempty closed convex subset of $\mathcal{H}$ and $T: C \rightarrow \mathcal{H}$ be a mapping. Then the following hold:

(i) $T$ is a nonexpansive mapping if and only if $I-T$ is $\frac{1}{2}$-inverse-strongly monotone $\left(\frac{1}{2}\right.$-ism).

(ii) If $S$ is $v$-ism, then $\gamma S$ is $\frac{v}{\gamma}$-ism.

(iii) $S$ is averaged if and only if $I-S$ is $v$-ism for some $v>\frac{1}{2}$.

Indeed, $S$ is $\alpha$-averaged if and only if $I-S$ is $\frac{1}{(2 \alpha)}$-ism for $\alpha \in(0,1)$.

(iv) If $S$ and $T$ are averaged, then the composition $S T$ is also averaged.

(v) If the mappings $\left\{T_{i}\right\}_{i=1}^{n}$ are averaged and have a common fixed point, then $\bigcap_{i=1}^{n} \operatorname{Fix}\left(T_{i}\right)=\operatorname{Fix}\left(T_{1} \cdots T_{n}\right)$ for each $n \in \mathbb{N}$.

Lemma 2.9 [30] Let T be a nonexpansive self-mapping on a nonempty closed convex subset $C$ of $\mathcal{H}$, and let $\left\{x_{n}\right\}$ be a sequence in $C$. If $x_{n} \rightarrow w$ and $\lim _{n \rightarrow \infty}\left\|x_{n}-T x_{n}\right\|=0$, then $T w=w$. 
Let $C$ be a nonempty closed convex subset of $\mathcal{H}$. The indicator function $\iota_{C}$ defined by

$$
\iota_{C} x= \begin{cases}0, & x \in C, \\ \emptyset, & x \notin C\end{cases}
$$

is a proper lower semicontinuous convex function and its subdifferential $\partial \iota_{C}$ defined by

$$
\partial \iota_{C} x=\left\{z \in \mathcal{H}:\langle y-x, z\rangle \leq \iota_{C}(y)-\iota_{C}(x), \forall y \in \mathcal{H}\right\}
$$

is a maximal monotone operator [31]. Furthermore, we also define the normal cone $N_{C} u$ of $C$ at $u$ as follows:

$$
N_{C} u=\{z \in \mathcal{H}:\langle z, v-u\rangle \leq 0, \forall v \in C\} .
$$

We can define the resolvent $J_{\lambda}^{\partial i_{C}}$ of $\partial i_{C}$ for $\lambda>0$, i.e.,

$$
J_{\lambda}^{\partial i_{C}} x=\left(I+\lambda \partial i_{C}\right)^{-1} x
$$

for all $x \in \mathcal{H}$. Since

$$
\partial i_{C} x=\left\{z \in \mathcal{H}: i_{C} x+\langle z, y-x\rangle \leq i_{C} y, \forall y \in \mathcal{H}\right\}=\{z \in \mathcal{H}:\langle z, y-x\rangle \leq 0, \forall y \in C\}=N_{C} x
$$

for all $x \in C$, we have that

$$
\begin{aligned}
u=J_{\lambda}^{\partial i_{C} x} & \Leftrightarrow \quad x \in u+\lambda \partial i_{C} u \\
& \Leftrightarrow \quad x-u \in \lambda N_{C} u \\
& \Leftrightarrow \quad\langle x-u, y-u\rangle \leq 0, \quad \forall y \in C \\
& \Leftrightarrow \quad u=P_{C} x .
\end{aligned}
$$

\section{Main results}

Let $C, Q$ and $Q^{\prime}$ be nonempty closed convex subsets of $\mathcal{H}_{1}, \mathcal{H}_{2}$ and $\mathcal{H}_{3}$, respectively. For each $i=1,2$ and $\kappa_{i}>0$, let $B_{i}$ be a $\kappa_{i}$-inverse-strongly monotone mapping of $C$ into $\mathcal{H}_{1}$, and let $G_{i}$ be a set-valued maximal monotone mapping on $\mathcal{H}_{1}$ such that the domain of $G_{i}$ is included in $C$ for each $i=1,2$. Let $F_{1}$ be a firmly nonexpansive mapping of $\mathcal{H}_{2}$ into $\mathcal{H}_{2}$ and $F_{2}$ be a firmly nonexpansive mapping of $\mathcal{H}_{3}$ into $\mathcal{H}_{3}$. Note $J_{\lambda}^{G_{1}}=\left(I+\lambda G_{1}\right)^{-1}$ and $J_{r}^{G_{2}}=$ $\left(I+r G_{2}\right)^{-1}$ for each $\lambda>0$ and $r>0$. Let $A_{1}: \mathcal{H}_{1} \rightarrow \mathcal{H}_{2}$ be a bounded linear operator, $A_{2}$ : $\mathcal{H}_{1} \rightarrow \mathcal{H}_{3}$ be a bounded linear operator, and $A_{i}^{*}$ be the adjoint of $A_{i}$ for $i=1,2$. Throughout this paper, we use these notations unless specified otherwise.

Theorem 3.1 Suppose that $\left(B_{1}+G_{1}\right)^{-1}(0) \cap\left(B_{2}+G_{2}\right)^{-1}(0)$ is nonempty, and $\left\{a_{n}\right\},\left\{b_{n}\right\},\left\{c_{n}\right\}$, $\left\{f_{n}\right\},\left\{g_{n}\right\},\left\{h_{n}\right\}$ are sequences in $[0,1]$ such that $a_{n}+b_{n}+c_{n}=1, f_{n}+g_{n}+h_{n}=1,0<a_{n}<1$, and $0<f_{n}<1$ for each $n \in \mathbb{N}$. For an arbitrarily fixed $u \in \mathcal{H}$, define a sequence $\left\{v_{n}\right\}$ by

$$
\left\{\begin{array}{l}
v_{0} \in \mathcal{H} \text { is chosen arbitrarily, } \\
v_{2 n+1}:=a_{n} u+b_{n} v_{2 n}+c_{n} J_{\delta_{n}}^{G_{1}}\left(I-\delta_{n} B_{1}\right) v_{2 n}, \quad n \in \mathbb{N} \cup\{0\} \\
v_{2 n}:=f_{n} u+g_{n} v_{2 n-1}+h_{n} J_{\gamma_{n}}^{G_{2}}\left(I-\gamma_{n} B_{2}\right) v_{2 n-1}, \quad n \in \mathbb{N} .
\end{array}\right.
$$


Then $\lim _{n \rightarrow \infty} v_{n}=P_{\left(B_{1}+G_{1}\right)^{-1}(0) \cap\left(B_{2}+G_{2}\right)^{-1}(0)}$ u provided the following conditions are satisfied:

(i) $\lim _{n \rightarrow \infty} a_{n}=\lim _{n \rightarrow \infty} f_{n}=0$;

(ii) either $\sum_{n=1}^{\infty} a_{n}=\infty$ or $\sum_{n=1}^{\infty} f_{n}=\infty$;

(iii) $0<a \leq \delta_{n} \leq b<2 \kappa_{1}$ and $0<f \leq \gamma_{n} \leq g<2 \kappa_{2}$ for each $n \in \mathbb{N}$ and for some $a, b, f, g \in \mathbb{R}^{+}$

(iv) $\liminf _{n \rightarrow \infty} c_{n}>0, \liminf _{n \rightarrow \infty} h_{n}>0$.

Proof Given any fixed $\bar{v}:=P_{\left(B_{1}+G_{1}\right)^{-1}(0) \cap\left(B_{2}+G_{2}\right)^{-1}(0)} u$. Then $\bar{v}=J_{\delta_{n}}^{G_{1}}\left(I-\delta_{n} B_{1}\right) \bar{v}$ and $\bar{v}=J_{\gamma_{n}}^{G_{2}}(I-$ $\left.\gamma_{n} B_{2}\right) \bar{v}$. Since $B_{1}$ is $\kappa_{1}$-ism and $\frac{\kappa_{1}}{\delta_{n}}>\frac{1}{2}$, it follows from Lemma 2.8 that $I-\delta_{n} B_{1}$ is averaged. Hence $I-\delta_{n} B_{1}$ is nonexpansive. By Lemma 2.8, $J_{\delta_{n}}^{G_{1}}\left(I-\delta_{n} G_{1}\right)$ and $J_{\gamma_{n}}^{G_{2}}\left(I-\gamma_{n} G_{2}\right)$ are averaged. Hence $J_{\delta_{n}}^{G_{1}}\left(I-\delta_{n} G_{1}\right)$ and $J_{\gamma_{n}}^{G_{2}}\left(I-\gamma_{n} G_{2}\right)$ are nonexpansive. By condition (iv), we may assume that there exist positive real numbers $c$ and $h$ such that $c_{n} \geq \ell>0$ and $h_{n} \geq h>0$ for each $n \in \mathbb{N}$. For each $n \in \mathbb{N}$, we have that

$$
\begin{aligned}
& \left\|v_{2 n}-\bar{v}\right\| \\
& \quad=\left\|f_{n} u+g_{n} v_{2 n-1}+h_{n} J_{\gamma_{n}}^{G_{2}}\left(I-\gamma_{n} B_{2}\right) v_{2 n-1}-\bar{v}\right\| \\
& \quad \leq f_{n}\|u-\bar{v}\|+g_{n}\left\|v_{2 n-1}-\bar{v}\right\|+h_{n}\left\|J_{\gamma_{n}}^{G_{2}}\left(I-\gamma_{n} B_{2}\right) v_{2 n-1}-\bar{v}\right\| \\
& \quad \leq f_{n}\|u-\bar{v}\|+g_{n}\left\|v_{2 n-1}-\bar{v}\right\|+h_{n}\left\|v_{2 n-1}-\bar{v}\right\| \\
& \quad=f_{n}\|u-\bar{v}\|+\left(g_{n}+h_{n}\right)\left\|v_{2 n-1}-\bar{v}\right\| \\
& \quad=f_{n}\|u-\bar{v}\|+\left(1-f_{n}\right)\left\|v_{2 n-1}-\bar{v}\right\|
\end{aligned}
$$

and

$$
\begin{aligned}
\left\|v_{2 n+1}-\bar{v}\right\| \\
\quad=\left\|a_{n} u+b_{n} v_{2 n}+c_{n} J_{\delta_{n}}^{G_{1}}\left(I-\delta_{n} B_{1}\right) v_{2 n}-\bar{v}\right\| \\
\quad \leq a_{n}\|u-\bar{v}\|+b_{n}\left\|v_{2 n}-\bar{v}\right\|+c_{n}\left\|J_{\delta_{n}}^{G_{1}}\left(I-\delta_{n} B_{1}\right) v_{2 n}-\bar{v}\right\| \\
\quad \leq a_{n}\|u-\bar{v}\|+b_{n}\left\|v_{2 n}-\bar{v}\right\|+c_{n}\left\|v_{2 n}-\bar{v}\right\| \\
\quad=a_{n}\|u-\bar{v}\|+\left(b_{n}+c_{n}\right)\left\|v_{2 n}-\bar{v}\right\| \\
\quad \leq\left(1-a_{n}\right)\left\{f_{n}\|u-\bar{v}\|+\left(1-f_{n}\right)\left\|v_{2 n-1}-\bar{v}\right\|\right\}+a_{n}\|u-\bar{v}\| \quad \text { by }(3.1) \\
\quad=f_{n}\left(1-a_{n}\right)\|u-\bar{v}\|+\left(\left(1-a_{n}\right)\left(1-f_{n}\right)\right)\left\|v_{2 n-1}-\bar{v}\right\|+a_{n}\|u-\bar{v}\| \\
\quad=\left(1-\left(1-a_{n}\right)\left(1-f_{n}\right)\right)\|u-\bar{v}\|+\left(1-a_{n}\right)\left(1-f_{n}\right)\left\|v_{2 n-1}-\bar{v}\right\| \\
\quad \leq \max \left\{\|u-\bar{v}\|,\left\|v_{2 n-1}-\bar{v}\right\|\right\} \\
\quad \leq \max \left\{\|u-\bar{v}\|,\left\|v_{1}-\bar{v}\right\|\right\} .
\end{aligned}
$$

By the mathematical induction method, we know that $\left\{v_{2 n-1}\right\},\left\{v_{2 n}\right\}$ and $\left\{v_{n}\right\}$ are bounded sequences. By Lemma 2.4, we have that

$$
\begin{aligned}
& \left\|J_{\delta_{n}}^{G_{1}}\left(I-\delta_{n} B_{1}\right) v_{2 n}-\bar{v}\right\|^{2} \\
& \quad=\left\|J_{\delta_{n}}^{G_{1}}\left(I-\delta_{n} B_{1}\right) v_{2 n}-J_{\delta_{n}}^{G_{1}}\left(I-\delta_{n} B_{1}\right) \bar{v}\right\|^{2} \\
& \quad \leq\left\|\left(I-\delta_{n} B_{1}\right) v_{2 n}-\left(I-\delta_{n} B_{1}\right) \bar{v}\right\|^{2}-\left\|\left(I-J_{\delta_{n}}^{G_{1}}\left(I-\delta_{n} B_{1}\right)\right) v_{2 n}-\left(I-J_{\delta_{n}}^{G_{1}}\left(I-\delta_{n} B_{1}\right)\right) \bar{v}\right\|^{2}
\end{aligned}
$$




$$
\begin{aligned}
& \leq\left\|v_{2 n}-\bar{v}\right\|^{2}-\left\|\left(I-J_{\delta_{n}}^{G_{1}}\left(I-\delta_{n} B_{1}\right)\right) v_{2 n}-\left(I-J_{\delta_{n}}^{G_{1}}\left(I-\delta_{n} B_{1}\right)\right) \bar{v}\right\|^{2} \\
& =\left\|v_{2 n}-\bar{v}\right\|^{2}-\left\|v_{2 n}-J_{\delta_{n}}^{G_{1}}\left(I-\delta_{n} B_{1}\right) v_{2 n}\right\|^{2}
\end{aligned}
$$

for each $n \in \mathbb{N}$. Hence,

$$
\begin{aligned}
\left\|v_{2 n+1}-\bar{v}\right\|^{2} & \\
= & \left\|a_{n}(u-\bar{v})+b_{n}\left(v_{2 n}-\bar{v}\right)+c_{n}\left(J_{\delta_{n}}^{G_{1}}\left(I-\delta_{n} B_{1}\right) v_{2 n}-\bar{v}\right)\right\|^{2} \\
\leq & \left\|b_{n}\left(v_{2 n}-\bar{v}\right)+c_{n}\left(J_{\delta_{n}}^{G_{1}}\left(I-\delta_{n} B_{1}\right) v_{2 n}-\bar{v}\right)\right\|^{2}+2 a_{n}\left\langle u-\bar{v}, v_{2 n+1}-\bar{v}\right\rangle \\
= & \left(1-a_{n}\right)^{2}\left\|b_{n}^{\prime}\left(v_{2 n}-\bar{v}\right)+c_{n}^{\prime}\left(J_{\delta_{n}}^{G_{1}}\left(I-\delta_{n} B_{1}\right) v_{2 n}-\bar{v}\right)\right\|^{2} \\
& +2 a_{n}\left\langle u-\bar{v}, v_{2 n+1}-\bar{v}\right\rangle \\
= & \left(1-a_{n}\right)^{2}\left\{b_{n}^{\prime}\left\|v_{2 n}-\bar{v}\right\|^{2}+c_{n}^{\prime}\left\|J_{\delta_{n}}^{G_{1}}\left(I-\delta_{n} B_{1}\right) v_{2 n}-\bar{v}\right\|^{2}\right. \\
& \left.-b_{n}^{\prime} c_{n}^{\prime}\left\|v_{2 n}-J_{\delta_{n}}^{G_{1}}\left(I-\delta_{n} B_{1}\right) v_{2 n}\right\|^{2}\right\}+2 a_{n}\left\langle u-\bar{v}, v_{2 n+1}-\bar{v}\right\rangle \\
\leq & b_{n}\left\|v_{2 n}-\bar{v}\right\|^{2}+c_{n}\left\|J_{\delta_{n}}^{G_{1}}\left(I-\delta_{n} B_{1}\right) v_{2 n}-\bar{v}\right\|^{2}+2 a_{n}\left\langle u-\bar{v}, v_{2 n+1}-\bar{v}\right\rangle \\
\leq & b_{n}\left\|v_{2 n}-\bar{v}\right\|^{2}+c_{n}\left(\left\|v_{2 n}-\bar{v}\right\|^{2}-\left\|v_{2 n}-J_{\delta_{n}}^{G_{1}}\left(I-\delta_{n} B_{1}\right) v_{2 n}\right\|^{2}\right)+2 a_{n}\left\langle u-\bar{v}, v_{2 n+1}-\bar{v}\right\rangle \\
= & \left(b_{n}+c_{n}\right)\left\|v_{2 n}-\bar{v}\right\|^{2}+2 a_{n}\left\langle u-\bar{v}, v_{2 n+1}-\bar{v}\right\rangle-c_{n}\left\|v_{2 n}-J_{\delta_{n}}^{G_{1}}\left(I-\delta_{n} B_{1}\right) v_{2 n}\right\|^{2}
\end{aligned}
$$

where

$$
b_{n}^{\prime}:=\frac{b_{n}}{b_{n}+c_{n}}, \quad c_{n}^{\prime}:=\frac{c_{n}}{b_{n}+c_{n}} .
$$

Similarly, we have

$$
\begin{aligned}
\left\|v_{2 n}-\bar{v}\right\|^{2} \leq & \left(g_{n}+h_{n}\right)\left\|v_{2 n-1}-\bar{v}\right\|^{2}+2 f_{n}\left\langle u-\bar{v}, v_{2 n}-\bar{v}\right\rangle \\
& -h_{n}\left\|J_{\gamma_{n}}^{G_{2}}\left(I-\gamma_{n} B_{2}\right) \nu_{2 n-1}-v_{2 n-1}\right\|^{2} .
\end{aligned}
$$

Consequently, it follows from (3.2) and (3.3) that

$$
\begin{aligned}
&\left\|v_{2 n+1}-\bar{v}\right\|^{2} \\
& \leq\left(b_{n}+c_{n}\right)\left\|v_{2 n}-\bar{v}\right\|^{2}+2 a_{n}\left\langle u-\bar{v}, v_{2 n+1}-\bar{v}\right\rangle-c_{n}\left\|J_{\delta_{n}}^{G_{1}}\left(I-\delta_{n} B_{1}\right) v_{2 n}-v_{2 n}\right\|^{2} \\
& \leq\left(1-a_{n}\right)\left\{\left(1-f_{n}\right)\left\|v_{2 n-1}-\bar{v}\right\|^{2}+2 f_{n}\left\langle u-\bar{v}, v_{2 n}-\bar{v}\right\rangle\right. \\
&\left.-h_{n}\left\|J_{\gamma_{n}}^{G_{2}}\left(I-\gamma_{n} B_{2}\right) v_{2 n-1}-v_{2 n-1}\right\|^{2}\right\}+2 a_{n}\left\langle u-\bar{v}, v_{2 n+1}-\bar{v}\right\rangle \\
&-c_{n}\left\|J_{\delta_{n}}^{G_{1}}\left(I-\delta_{n} B_{1}\right) v_{2 n}-v_{2 n}\right\|^{2} \\
&=\left(1-a_{n}\right)\left(1-f_{n}\right)\left\|v_{2 n-1}-\bar{v}\right\|^{2}+2 f_{n}\left(1-a_{n}\right)\left\langle u-\bar{v}, v_{2 n}-\bar{v}\right\rangle \\
&-h_{n}\left(1-a_{n}\right)\left\|J_{\gamma_{n}}^{G_{2}}\left(I-\gamma_{n} B_{2}\right) v_{2 n-1}-v_{2 n-1}\right\|^{2}+2 a_{n}\left\langle u-\bar{v}, v_{2 n+1}-\bar{v}\right\rangle \\
&-c_{n}\left\|J_{\delta_{n}}^{G_{1}}\left(I-\delta_{n} B_{1}\right) v_{2 n}-v_{2 n}\right\|^{2} .
\end{aligned}
$$


For each $n \in \mathbb{N}$, set $S_{n}:=\left\|v_{2 n-1}-\bar{v}\right\|^{2}$. Then $S_{n+1}=\left\|v_{2 n+1}-\bar{v}\right\|^{2}$ and (3.4) become

$$
\begin{aligned}
S_{n+1} \leq & \left(1-a_{n}\right)\left(1-f_{n}\right) S_{n}+2 a_{n}\left\langle u-\bar{v}, v_{2 n+1}-\bar{v}\right\rangle+2 f_{n}\left(1-a_{n}\right)\left\langle u-\bar{v}, v_{2 n}-\bar{v}\right\rangle \\
& -c_{n}\left\|J_{\delta_{n}}^{G_{1}}\left(I-\delta_{n} B_{1}\right) v_{2 n}-v_{2 n}\right\|^{2} \\
& -h_{n}\left(1-a_{n}\right)\left\|J_{\gamma_{n}}^{G_{2}}\left(I-\gamma_{n} B_{2}\right) v_{2 n-1}-v_{2 n-1}\right\|^{2} \\
\leq & \left(1-a_{n}\right)\left(1-f_{n}\right) S_{n}+2 a_{n}\left\langle u-\bar{v}, v_{2 n+1}-\bar{v}\right\rangle+2 f_{n}\left(1-a_{n}\right)\left\langle u-\bar{v}, v_{2 n}-\bar{v}\right\rangle .
\end{aligned}
$$

Case 1: $\left\{S_{n}\right\}$ is eventually decreasing, i.e., there exists a natural number $N$ such that $\left\|v_{2 n+1}-\bar{v}\right\| \leq\left\|v_{2 n-1}-\bar{v}\right\|$ for each $n \geq N$. So, $\left\{S_{n}\right\}$ is convergent and $\lim _{n \rightarrow \infty}\left\|v_{2 n-1}-\bar{v}\right\|$ exists. For all $n \in \mathbb{N}, c_{n} \geq c, h_{n} \geq h$ and (3.5), we have that

$$
\begin{aligned}
0 \leq & c\left\|J_{\delta_{n}}^{G_{1}}\left(I-\delta_{n} B_{1}\right) v_{2 n}-v_{2 n}\right\|^{2} \\
& +h\left(1-a_{n}\right)\left\|J_{\gamma_{n}}^{G_{2}}\left(I-\gamma_{n} B_{2}\right) v_{2 n-1}-v_{2 n-1}\right\|^{2} \\
\leq & \left(1-a_{n}\right)\left(1-f_{n}\right) S_{n}-S_{n+1}+2 a_{n}\left\langle u-\bar{v}, v_{2 n+1}-\bar{v}\right\rangle+2 f_{n}\left(1-a_{n}\right)\left\langle u-\bar{v}, v_{2 n}-\bar{v}\right\rangle .
\end{aligned}
$$

Noting via condition (i) and the fact that $\left\{v_{n}\right\}$ is bounded that

$$
\begin{aligned}
& \lim _{n \rightarrow \infty}\left[\left(1-a_{n}\right)\left(1-f_{n}\right) S_{n}-S_{n+1}\right]=0, \\
& \lim _{n \rightarrow \infty} 2 a_{n}\left\langle u-\bar{v}, v_{2 n+1}-\bar{v}\right\rangle=\lim _{n \rightarrow \infty} 2 f_{n}\left(1-a_{n}\right)\left\langle u-\bar{v}, v_{2 n}-\bar{v}\right\rangle=0,
\end{aligned}
$$

we conclude from (3.6) that

$$
\lim _{n \rightarrow \infty}\left(c\left\|J_{\delta_{n}}^{G_{1}}\left(I-\delta_{n} B_{1}\right) v_{2 n}-v_{2 n}\right\|^{2}+h\left(1-a_{n}\right)\left\|J_{\gamma_{n}}^{G_{2}}\left(I-\gamma_{n} B_{2}\right) v_{2 n-1}-v_{2 n-1}\right\|^{2}\right)=0
$$

Therefore,

$$
\lim _{n \rightarrow \infty}\left\|J_{\delta_{n}}^{G_{1}}\left(I-\delta_{n} B_{1}\right) v_{2 n}-v_{2 n}\right\|=\lim _{n \rightarrow \infty}\left\|J_{\gamma_{n}}^{G_{2}}\left(I-\gamma_{n} B_{2}\right) v_{2 n-1}-v_{2 n-1}\right\|=0
$$

Since $\left\{v_{2 n}\right\}$ is a bounded sequence in $\mathcal{H}$, there is a subsequence $\left\{v_{(2 n)_{k}}\right\}$ of $\left\{v_{2 n}\right\}$ such that $v_{(2 n)_{k}} \rightarrow \bar{x} \in \mathcal{H}$ and

$$
\limsup _{n \rightarrow \infty}\left\langle u-\bar{v}, v_{2 n}-\bar{v}\right\rangle=\lim _{k \rightarrow \infty}\left\langle u-\bar{v}, v_{(2 n)_{k}}-\bar{v}\right\rangle=\langle u-\bar{v}, \bar{x}-\bar{v}\rangle
$$

On the other hand, $0<a \leq \delta_{n} \leq b<2 \kappa_{1}$, there exists a subsequence $\left\{\delta_{n_{k_{j}}}\right\}$ of $\left\{\delta_{n}\right\}$ such that $\left\{\delta_{n_{k_{j}}}\right\}$ converges to a number $\bar{\delta} \in[a, b]$. By Lemma 2.5, we have

$$
\begin{aligned}
& \left\|v_{(2 n)_{k_{j}}}-J_{\bar{\delta}}^{G_{1}}\left(I-\bar{\delta} B_{1}\right) v_{(2 n)_{k_{j}}}\right\| \\
& \leq\left\|v_{(2 n)_{k_{j}}}-J_{\delta_{n_{k_{j}}}}^{G_{1}}\left(I-\delta_{n_{k_{j}}} B_{1}\right) v_{(2 n)_{k_{j}}}\right\| \\
& \quad+\left\|J_{\delta_{n_{k_{j}}}}^{G_{1}}\left(I-\bar{\delta} B_{1}\right) v_{(2 n)_{k_{j}}}-J_{\bar{\delta}}^{G_{1}}\left(I-\bar{\delta} B_{1}\right) v_{(2 n)_{k_{j}}}\right\| \\
& \quad+\left\|J_{\delta_{n_{k_{j}}}}^{G_{1}}\left(I-\delta_{n_{k_{j}}} B_{1}\right) v_{(2 n)_{k_{j}}}-J_{\delta_{n_{k_{j}}}}^{G_{1}}\left(I-\bar{\delta} B_{1}\right) v_{(2 n)_{k_{j}}}\right\|
\end{aligned}
$$




$$
\begin{aligned}
\leq & \left\|v_{(2 n)_{k_{j}}}-J_{\delta_{n_{k_{j}}}}^{G_{1}}\left(I-\delta_{n_{k_{j}}} B_{1}\right) v_{(2 n)_{k_{j}}}\right\|+\left|\delta_{n_{k_{j}}}-\bar{\delta}\right|\left\|B_{1} u_{n_{k_{j}}}\right\| \\
& +\frac{\left|\delta_{n_{k_{j}}}-\bar{\delta}\right|}{\bar{\delta}}\left\|J_{\bar{\delta}}^{G_{1}}\left(I-\bar{\delta} B_{1}\right) v_{(2 n)_{k_{j}}}-\left(I-\bar{\delta} B_{1}\right) v_{(2 n)_{k_{j}}}\right\| \rightarrow 0 .
\end{aligned}
$$

By (3.8) and Lemma 2.9, $\bar{x} \in \operatorname{Fix}\left(J_{\bar{\delta}}^{G_{1}}\left(I-\bar{\delta} B_{1}\right)\right)=\left(B_{1}+G_{1}\right)^{-1}(0)$. Since $0<c \leq \gamma_{n} \leq d<2 \kappa_{2}$, there exists a subsequence $\left\{\gamma_{n_{k_{j}}}\right\}$ of $\left\{\gamma_{n}\right\}$ such that $\left\{\gamma_{n_{k_{j}}}\right\}$ converges to a number $\bar{\gamma} \in[c, d]$. We have that

$$
\begin{aligned}
\left\|v_{2 n+1}-v_{2 n}\right\| & =\left\|a_{n} u+b_{n} v_{2 n}+c_{n} J_{\delta_{n}}^{G_{1}}\left(I-\delta_{n} B_{1}\right) v_{2 n}-v_{2 n}\right\| \\
& \leq a_{n}\left\|u-v_{2 n}\right\|+c_{n}\left\|J_{\delta_{n}}^{G_{1}}\left(I-\delta_{n} B_{1}\right) v_{2 n}-v_{2 n}\right\|
\end{aligned}
$$

and

$$
\begin{aligned}
\left\|v_{2 n}-v_{2 n-1}\right\| & =\left\|f_{n} u+g_{n} v_{2 n-1}+h_{n} J_{\gamma_{n}}^{G_{2}}\left(I-\gamma_{n} B_{2}\right) v_{2 n-1}-v_{2 n-1}\right\| \\
& \leq f_{n}\left\|u-v_{2 n-1}\right\|+h_{n}\left\|J_{\gamma_{n}}^{G_{2}}\left(I-\gamma_{n} B_{2}\right) v_{2 n-1}-v_{2 n-1}\right\| .
\end{aligned}
$$

Since $\left\{v_{n}\right\}$ is bounded, we conclude from (3.7), (3.9), (3.10), and conditions (i), (ii) that

$$
\lim _{n \rightarrow \infty}\left\|v_{n+1}-v_{n}\right\|=0 .
$$

By Lemma 2.5, we have

$$
\begin{aligned}
\left\|v_{(2 n)_{k_{j}}+1}-J_{\bar{\gamma}}\left(I-\bar{\gamma} B_{2}\right) v_{(2 n)_{k_{j}}+1}\right\| \\
\leq\left\|v_{(2 n)_{k_{j}}+1}-J_{\gamma_{n_{k_{j}}}}\left(I-\gamma_{n_{k_{j}}} B_{2}\right) v_{(2 n)_{k_{j}}+1}\right\| \\
\quad+\left\|J_{\gamma_{n_{k_{j}}}}\left(I-\gamma_{n_{k_{j}}} B_{2}\right) v_{(2 n)_{k_{j}}+1}-J_{\gamma_{n_{k_{j}}}}\left(I-\bar{\gamma} B_{2}\right) v_{(2 n)_{k_{j}}+1}\right\| \\
\quad+\left\|J_{\gamma_{n_{k_{j}}}}\left(I-\bar{\gamma} B_{2}\right) v_{(2 n)_{k_{j}}+1}-J_{\bar{r}}\left(I-\bar{r} B_{2}\right) v_{(2 n)_{k_{j}}+1}\right\| \\
\leq\left\|v_{(2 n)_{k_{j}}+1}-J_{\gamma_{n_{k_{j}}}}\left(I-\gamma_{n_{k_{j}}} B_{2}\right) v_{(2 n)_{k_{j}}+1}\right\|+\left|\gamma_{n_{k_{j}}}-\bar{\gamma}\right|\left\|B_{2} v_{(2 n)_{k_{j}}+1}\right\| \\
\quad+\frac{\left|\gamma_{n_{k_{j}}}-\bar{\gamma}\right|}{\bar{\gamma}}\left\|J_{\bar{\gamma}}\left(I-\bar{\gamma} B_{2}\right) v_{(2 n)_{k_{j}}+1}-\left(I-\bar{\gamma} B_{2}\right) v_{(2 n)_{k_{j}}+1}\right\| \rightarrow 0 .
\end{aligned}
$$

Since $\lim _{n \rightarrow \infty}\left\|v_{2 n+1}-v_{2 n}\right\|=0$, we know that $v_{(2 n)_{k_{j}+1}} \rightarrow \bar{x}$. By (3.12) and Lemma 2.9, we know that $J_{\bar{\gamma}}^{G_{2}}\left(I-\bar{\gamma} B_{2}\right) \bar{x}=\bar{x}$. So, $\bar{x} \in\left(B_{2}+G_{2}\right)^{-1}(0)$. This shows that $\bar{x} \in\left(B_{1}+G_{1}\right)^{-1}(0) \cap$ $\left(B_{2}+G_{2}\right)^{-1}(0)$. It follows from $\bar{v}:=P_{\left(B_{1}+G_{1}\right)^{-1}(0) \cap\left(B_{2}+G_{2}\right)^{-1}(0)} u$ and Lemma 2.3 that

$$
\limsup _{n \rightarrow \infty}\left\langle u-\bar{v}, v_{2 n}-\bar{v}\right\rangle=\langle u-\bar{v}, \bar{x}-\bar{v}\rangle \leq 0 .
$$

By (3.11) and (3.13),

$$
\begin{aligned}
& \limsup _{n \rightarrow \infty}\left\langle u-\bar{v}, v_{2 n+1}-\bar{v}\right\rangle \\
& \quad=\limsup _{n \rightarrow \infty}\left(\left\langle u-\bar{v}, v_{2 n+1}-v_{2 n}\right\rangle+\left\langle u-\bar{v}, v_{2 n}-\bar{v}\right\rangle\right) \\
& \quad \leq \limsup _{n \rightarrow \infty}\left\langle u-\bar{v}, v_{2 n+1}-v_{2 n}\right\rangle+\limsup _{n \rightarrow \infty}\left\langle u-\bar{v}, v_{2 n}-\bar{v}\right\rangle \leq 0 .
\end{aligned}
$$


Applying Lemma 2.7 to inequality (3.5) with $t_{n}=2\left\langle u-\bar{v}, v_{2 n+1}-\bar{v}\right\rangle$ and $k_{n}=2\left(1-a_{n}\right)\langle u-$ $\left.\bar{v}, v_{2 n}-\bar{v}\right\rangle$, we obtain from (3.13) and (3.14) and conditions (i), (ii) that $\lim _{n \rightarrow \infty} S_{n}=0$. That is, $\lim _{n \rightarrow \infty} v_{2 n-1}=\bar{v}$. And then it follows from (3.11) that $\lim _{n \rightarrow \infty} v_{2 n}=\bar{v}$. Thus, $\lim _{n \rightarrow \infty} v_{n}=\bar{v}$.

Case 2: Suppose that $\left\{S_{n}\right\}$ is not an eventually decreasing sequence. Let $\left\{S_{n_{i}}\right\}$ be a subsequence of $\left\{S_{n}\right\}$ such that $S_{n_{i}} \leq S_{n_{i}+1}$ for all $i \geq 0$, also consider the sequence of integers $\{\tau(n)\}_{n \geq n_{0}}$, defined by $\tau(n)=\max \left\{k \leq n, S_{k}<S_{k+1}\right\}$, for some $n_{0}$ ( $n_{0}$ is a sufficiently large number). Then $\{\tau(n)\}_{n \geq n_{0}}$ is a nondecreasing sequence as $\lim _{n \rightarrow \infty} \tau(n)=\infty$, and for all $n \geq n_{0}$, one has that

$$
S_{\tau(n)} \leq S_{\tau(n)+1} \quad \text { and } \quad S_{n} \leq S_{\tau(n)+1} .
$$

That is, $\max \left\{S_{\tau(n)}, S_{n}\right\} \leq S_{\tau(n)+1}$. For such $n \geq n_{0}$, it follows from (3.15) that

$$
\left\|v_{2 \tau(n)-1}-\bar{v}\right\| \leq\left\|v_{2 \tau(n)+1}-\bar{v}\right\|
$$

and

$$
\left\|v_{2 n-1}-\bar{v}\right\| \leq\left\|v_{2 \tau(n)+1}-\bar{v}\right\|
$$

From (3.15) and (3.5), we obtain

$$
\begin{aligned}
S_{\tau(n)} \leq & S_{\tau(n)+1} \\
\leq & \left(1-a_{\tau(n)}\right)\left(1-f_{\tau(n)}\right) S_{\tau(n)}+2 a_{\tau(n)}\left\langle u-\bar{v}, v_{2 \tau(n)+1}-\bar{v}\right\rangle \\
& +2 f_{\tau(n)}\left(1-a_{\tau(n)}\right)\left\langle u-\bar{v}, v_{2 \tau(n)}-\bar{v}\right\rangle \\
& -c_{\tau(n)}\left\|J_{\delta_{\tau(n)}}^{G_{1}}\left(I-\delta_{\tau(n)} B_{1}\right) v_{2 \tau(n)}-v_{2 \tau(n)}\right\|^{2} \\
& -h_{\tau(n)}\left\|J_{\gamma_{\tau(n)}}^{G_{2}}\left(I-\gamma_{\tau(n)} B_{2}\right) v_{2 \tau(n)-1}-v_{2 \tau(n)-1}\right\|^{2} \\
\leq & \left(1-a_{\tau(n)}\right)\left(1-f_{\tau(n)}\right) S_{\tau(n)}+2 a_{\tau(n)}\left\langle u-\bar{v}, v_{2 \tau(n)+1}-\bar{v}\right\rangle \\
& +2 f_{\tau(n)}\left(1-a_{\tau(n)}\right)\left\langle u-\bar{v}, v_{2 \tau(n)}-\bar{v}\right\rangle .
\end{aligned}
$$

Just as the argument of Case 1, we have

$$
\left\{\begin{array}{l}
\lim _{n \rightarrow \infty}\left\|v_{2 \tau(n)}-J_{\delta_{\tau(n)}}^{G_{1}}\left(I-\delta_{\tau(n)} B_{1}\right) v_{2 \tau(n)}\right\|=0, \\
\lim _{n \rightarrow \infty}\left\|v_{2 \tau(n)-1}-J_{\gamma_{\tau}(n)}^{G_{2}}\left(I-\gamma_{\tau(n)} B_{2}\right) v_{2 \tau(n)-1}\right\|=0, \\
\limsup _{n \rightarrow \infty}\left\langle u-\bar{v}, v_{2 \tau(n)+1}-\bar{v}\right\rangle \leq 0, \\
\limsup _{n \rightarrow \infty}\left\langle u-\bar{v}, v_{2 \tau(n)}-\bar{v}\right\rangle \leq 0, \\
\lim _{n \rightarrow \infty}\left\|v_{2 \tau(n)+1}-v_{2 \tau(n)}\right\|=0 .
\end{array}\right.
$$

By (3.15) and (3.18), we have

$$
\begin{aligned}
S_{\tau(n)+1} \leq & \left(1-a_{\tau(n)}\right)\left(1-f_{\tau(n)}\right) S_{\tau(n)+1}+2 a_{\tau(n)}\left\langle u-\bar{v}, v_{2 \tau(n)+1}-\bar{v}\right\rangle \\
& +2 f_{\tau(n)}\left(1-a_{\tau(n)}\right)\left\langle u-\bar{v}, v_{2 \tau(n)}-\bar{v}\right\rangle
\end{aligned}
$$


for all $n \geq n_{0}$. This implies that

$$
\begin{aligned}
S_{\tau(n)+1} \leq & \left(1-a_{\tau(n)}\right)\left(1-f_{\tau(n)}\right) S_{\tau(n)+1}+a_{\tau(n)} K\left\|v_{2 \tau(n)+1}-v_{2 \tau(n)}\right\| \\
& +2\left[f_{\tau(n)}\left(1-a_{\tau(n)}\right)+a_{\tau(n)}\right]\left\langle u-\bar{v}, v_{2 \tau(n)}-\bar{v}\right\rangle
\end{aligned}
$$

for all $n \geq n_{0}$, where $K=2\|u-\bar{v}\|$. Furthermore, we have

$$
\begin{aligned}
S_{\tau(n)+1} \leq & \frac{a_{\tau(n)} K\left\|v_{2 \tau(n)+1}-v_{2 \tau(n)}\right\|}{a_{\tau(n)}+f_{\tau(n)}\left(1-a_{\tau(n)}\right)} \\
& +2\left\langle u-\bar{v}, v_{2 \tau(n)}-\bar{v}\right\rangle \\
\leq & K\left\|v_{2 \tau(n)+1}-v_{2 \tau(n)}\right\|+2\left\langle u-\bar{v}, v_{2 \tau(n)}-\bar{v}\right\rangle .
\end{aligned}
$$

Hence, it follows from (3.19) and (3.20) that

$$
\lim _{n \rightarrow \infty} S_{\tau(n)+1}=0 .
$$

By (3.15) and (3.21), we know that $\lim _{n \rightarrow \infty} S_{n}=0$. Then, just as the argument in the proof of Case 1, we obtain $\lim _{n \rightarrow \infty} v_{n}=\bar{v}$. Therefore, the proof is completed.

\section{Remark 3.1}

(i) If we put $B_{1}=B_{2}=0$ in Theorem 3.1, then Theorem 3.1 is reduced to Theorem 7 in [20].

(ii) Boikanyo et al. [20] showed that strong convergence theorem of a proximal point algorithm with error can be obtained from strong convergence of a proximal point algorithm without errors. Therefore, in Theorem 3.1, we study strong convergence of variational inclusion problems without error.

As a simple consequence of Theorem 3.1, we have the following theorem.

Theorem 3.2 Suppose that $\left(B_{1}+G_{1}\right)^{-1}(0)$ is nonempty, and $\left\{a_{n}\right\},\left\{b_{n}\right\},\left\{c_{n}\right\}$ are sequences in $[0,1]$ such that $a_{n}+b_{n}+c_{n}=1,0<a_{n}<1$ for each $n \in \mathbb{N}$. For an arbitrary fixed $u \in \mathcal{H}$, define a sequence $\left\{x_{n}\right\}$ by

$$
\left\{\begin{array}{l}
x_{0} \in \mathcal{H} \text { is chosen arbitrarily, } \\
x_{n+1}:=a_{n} u+b_{n} x_{n}+c_{n} J_{\delta_{n}}^{G_{1}}\left(I-\delta_{n} B_{1}\right) x_{n}, \quad n \in \mathbb{N} \cup\{0\}
\end{array}\right.
$$

Then $\lim _{n \rightarrow \infty} v_{n}=P_{\left(B_{1}+G_{1}\right)^{-1}(0)} u$ if the following conditions are satisfied:

(i) $\lim _{n \rightarrow \infty} a_{n}=0, \sum_{n=1}^{\infty} a_{n}=\infty$;

(ii) $0<a \leq \delta_{n} \leq b<2 \kappa_{1}$ for each $n \in \mathbb{N}$ and for some $a, b \in \mathbb{R}^{+}$;

(iii) $\liminf _{n \rightarrow \infty} c_{n}>0$.

Proof Set $f_{n}=0, B_{2}=0, g_{n}+h_{n}=1,\left\{g_{n}\right\}$ and $\left\{h_{n}\right\}$ are sequences in $[0,1]$, and $G_{2}=\partial \iota_{\mathcal{H}_{1}}$. Define a sequence $v_{n}$ by

$$
v_{2 n+1}=a_{n} u+b_{n} x_{n}+c_{n} J_{\delta_{n}}^{G_{1}}\left(I-\delta_{n} B_{1}\right) x_{n}, \quad n \in \mathbb{N} \cup\{0\}
$$


and

$$
v_{2 n}=v_{2 n-1}=x_{n-1} .
$$

Then

$$
v_{2 n+1}=a_{n} u+b_{n} v_{2 n}+c_{n} J_{\delta_{n}}^{G_{1}}\left(I-\delta_{n} B_{1}\right) \nu_{2 n}, \quad n \in \mathbb{N} \cup\{0\}
$$

and

$$
v_{2 n}=f_{n} u+g_{n} v_{2 n-1}+h_{n} v_{2 n-1}=f_{n} u+g_{n} v_{2 n-1}+h_{n} J_{\gamma_{n}}^{\partial \iota_{\mathcal{H}}} v_{2 n-1}, \quad n \in \mathbb{N} \cup\{0\} .
$$

Since

$$
G_{2}^{-1}(0)=\left(\partial \iota_{\mathcal{H}_{1}}\right)^{-1} 0=\operatorname{Fix}\left(J_{\gamma_{n}}^{\partial \iota \mathcal{H}_{1}}\right)=\operatorname{Fix}\left(P_{\mathcal{H}_{1}}\right)=\mathcal{H}_{1}
$$

it is easy to see that

$$
\left(B_{1}+G_{1}\right)^{-1}(0)=\left(\left(B_{1}+G_{1}\right)^{-1}(0) \cap \mathcal{H}_{1}\right)=\left(\left(B_{1}+G_{1}\right)^{-1}(0) \cap\left(B_{2}+G_{2}\right)^{-1}(0)\right) \neq \emptyset .
$$

Then Theorem 3.2 follows from Theorem 3.1.

Remark 3.2 Following the same argument as in Remark 12 in [20], we see that Theorems 3.1 and 3.2 contain [23, Theorem 3.3], [24, Theorem 1], [32, Theorems 1-4] and many other recent results as special cases.

\section{Applications}

Now, we recall the following multiple sets split feasibility problem (MSSFP-A1):

Find $\bar{x} \in \mathcal{H}_{1}$ such that $\bar{x} \in G_{1}^{-1}(0) \cap G_{2}^{-1}(0), A_{1} \bar{x} \in \operatorname{Fix}\left(F_{1}\right)$ and $A_{2} \bar{x} \in \operatorname{Fix}\left(F_{2}\right)$.

Theorem 4.1 [33] Given any $\bar{x} \in \mathcal{H}_{1},\left\{\lambda_{n}\right\}$ and $\left\{\gamma_{n}\right\}$ are sequences in $(0, \infty)$.

(i) If $\bar{x}$ is a solution of (MSSFP-A1), then $J_{\lambda_{n}}^{G_{1}}\left(I-\rho_{n} A_{1}^{*}\left(I-F_{1}\right) A_{1}\right) J_{r_{n}}^{G_{2}}\left(I-\sigma_{n} A_{2}^{*}\left(I-F_{2}\right) A_{2}\right) \bar{x}=\bar{x}$ for each $n \in \mathbb{N}$.

(ii) Suppose that $J_{\lambda_{n}}^{G_{1}}\left(I-\rho_{n} A_{1}^{*}\left(I-F_{1}\right) A_{1}\right) J_{r_{n}}^{G_{2}}\left(I-\sigma_{n} A_{2}^{*}\left(I-F_{2}\right) A_{2}\right) \bar{x}=\bar{x}$ with $0<\rho_{n}<\frac{2}{\left\|A_{1}\right\|^{2}+2}, 0<\sigma_{n}<\frac{2}{\left\|A_{2}\right\|^{2}+2}$ for each $n \in \mathbb{N}$ and the solution set of (MSSFP-A1) is nonempty. Then $\bar{x}$ is a solution of (MSSFP-A1).

In order to study the convergence theorems for the solution set of multiple split feasibility problem (MSSFP-A1), we need the following problems and the following essential tool which is a special case of Theorem 3.2 in [33]:

(SFP-1) Find $\bar{x} \in \mathcal{H}_{1}$ such that $\bar{x} \in \operatorname{Fix}\left(J_{\rho_{n}}^{G_{1}}\right)$ and $A_{1} \bar{x} \in \operatorname{Fix}\left(F_{1}\right)$.

Lemma 4.1 Given any $\bar{x} \in \mathcal{H}_{1}$.

(i) If $\bar{x}$ is a solution of (SFP-1), then $J_{\rho_{n}}^{G_{1}}\left(I-\rho_{n} A_{1}^{*}\left(I-F_{1}\right) A_{1}\right) \bar{x}=\bar{x}$ for each $n \in \mathbb{N}$. 
(ii) Suppose that $J_{\rho_{n}}^{G_{1}}\left(I-\rho_{n} A_{1}^{*}\left(I-F_{1}\right) A_{1}\right) \bar{x}=\bar{x}$ with $0<\rho_{n}<\frac{2}{\left\|A_{1}\right\|^{2}+2}$ for each $n \in \mathbb{N}$, and the solution set of (SFP-1) is nonempty. Then $\left(I-\rho_{n} A_{1}^{*}\left(I-F_{1}\right) A_{1}\right)$ and $J_{\rho_{n}}^{G_{1}}\left(I-\rho_{n} A_{1}^{*}\left(I-F_{1}\right) A_{1}\right)$ are averaged and $\bar{x}$ is a solution of (SFP-1).

Proof (i) Suppose that $\bar{x} \in \mathcal{H}_{1}$ is a solution of (SFP-1). Then $\bar{x} \in \operatorname{Fix}\left(J_{\rho_{n}}^{G_{1}}\right), A_{1} \bar{x} \in \operatorname{Fix}\left(F_{1}\right)$ for each $n \in \mathbb{N}$. It is easy to see that

$$
J_{\rho_{n}}^{G_{1}}\left(I-\rho_{n} A_{1}^{*}\left(I-F_{1}\right) A_{1}\right) \bar{x}=\bar{x}, \quad n \in \mathbb{N} .
$$

(ii) Since the solution set of (SFP-1) is nonempty, there exists $\bar{w} \in \mathcal{H}_{1}$ such that $\bar{w} \in$ $\operatorname{Fix}\left(J_{\rho_{n}}^{G_{1}}\right), A_{1} \bar{w} \in \operatorname{Fix}\left(F_{1}\right)$. Then $\bar{w} \in G_{1}^{-1}(0)$. If we put $G_{2}=G_{1}$ and $F_{2}=F_{1}$, we get that the solution set of (MSSFP-A1) is nonempty. By Lemma 2.1 we have that

$$
A_{1}^{*}\left(I-F_{1}\right) A_{1} \text { is } \frac{1}{\left\|A_{1}\right\|^{2}} \text {-ism. }
$$

By (4.1), $0<\rho_{n}<\frac{2}{\left\|A_{1}\right\|^{2}+2}$, and Lemma 2.8(ii), (iii), we know that

$$
I-\rho_{n} A_{1}^{*}\left(I-F_{1}\right) A_{1} \text { is averaged for each } n \in \mathbb{N} \text {. }
$$

On the other hand, for each $n \in \mathbb{N}, J_{\rho_{n}}^{G_{1}}$ is a firmly nonexpansive mappings, it is easy to see that

$$
J_{\rho_{n}}^{G_{1}} \text { is } \frac{1}{2} \text {-averaged. }
$$

Hence, by (4.2), (4.3) and Lemma 2.8(iv) and (v), we see that

$$
J_{\rho_{n}}^{G_{1}}\left(I-\rho_{n} A_{1}^{*}\left(I-F_{1}\right) A_{1}\right) \text { is averaged. }
$$

Since

$$
J_{\rho_{n}}^{G_{1}}\left(I-\rho_{n} A_{1}^{*}\left(I-F_{1}\right) A_{1}\right) \bar{x}=\bar{x},
$$

so

$$
J_{\rho_{n}}^{G_{1}}\left(I-\rho_{n} A_{1}^{*}\left(I-F_{1}\right) A_{1}\right) J_{\rho_{n}}^{G_{1}}\left(I-\rho_{n} A_{1}^{*}\left(I-F_{1}\right) A_{1}\right) \bar{x}=\bar{x} .
$$

Then Lemma 4.1 follows from Theorem 4.1 by taking $G_{1}=G_{2}, F_{1}=F_{2}$ and $\rho_{n}=r_{n}$.

Remark 4.1 From the following result, we know that Lemma 4.1 is more useful than Theorem 4.1 .

Theorem 4.2 Suppose that the solution set $\Omega_{A 1}$ of (MSSFP-A1) is nonempty and $\left\{a_{n}\right\},\left\{b_{n}\right\}$, $\left\{c_{n}\right\},\left\{f_{n}\right\},\left\{g_{n}\right\},\left\{h_{n}\right\}$ are sequences in $[0,1]$ such that $a_{n}+b_{n}+c_{n}=1, f_{n}+g_{n}+h_{n}=1,0<a_{n}<1$, and $0<f_{n}<1$ for each $n \in \mathbb{N}$. For an arbitrarily fixed $u \in \mathcal{H}$, a sequence $\left\{v_{n}\right\}$ is defined by

$$
\left\{\begin{array}{l}
v_{0} \in \mathcal{H} \text { is chosen arbitrarily, } \\
v_{2 n+1}:=a_{n} u+b_{n} v_{2 n}+c_{n} J_{\rho_{n}}\left(I-\rho_{n} A_{1}^{*}\left(I-F_{1}\right) A_{1}\right) v_{2 n}, \quad n \in \mathbb{N} \cup\{0\}, \\
v_{2 n}:=f_{n} u+g_{n} v_{2 n-1}+h_{n} J_{\sigma_{n}}^{G_{2}}\left(I-\sigma_{n} A_{2}^{*}\left(I-F_{2}\right) A_{2}\right) v_{2 n-1}, \quad n \in \mathbb{N} .
\end{array}\right.
$$


Then $\lim _{n \rightarrow \infty} v_{n}$ is a unique solution of the following optimization problem:

$$
\min _{q \in \Omega_{A 1}}\|q-u\|
$$

provided the following conditions are satisfied:

(i) $\lim _{n \rightarrow \infty} a_{n}=\lim _{n \rightarrow \infty} f_{n}=0$;

(ii) either $\sum_{n=1}^{\infty} a_{n}=\infty$ or $\sum_{n=1}^{\infty} f_{n}=\infty$;

(iii) $0<a \leq \rho_{n} \leq b<\frac{2}{\left\|A_{1}\right\|^{2}+2}, 0<c \leq \sigma_{n} \leq d<\frac{2}{\left\|A_{2}\right\|^{2}+2}$ for each $n \in \mathbb{N}$, and for some $a, b, c, d \in \mathbb{R}^{+}$

(iv) $\liminf _{n \rightarrow \infty} c_{n}>0, \liminf _{n \rightarrow \infty} h_{n}>0$.

Proof Since $F_{i}$ is firmly nonexpansive, it follows from Lemma 4.1 that $A_{i}^{*}\left(I-F_{i}\right) A_{i}$ is $\frac{1}{\left\|A_{i}\right\|^{2}}$ ism for each $i=1,2$. For each $i=1,2$, put $B_{i}=A_{i}^{*}\left(I-F_{i}\right) A_{i}$ in Lemma 4.1. Then algorithm in Theorem 3.1 follows immediately from algorithm in Theorem 4.2. Since $\Omega_{A 1}$ is nonempty, by Lemma 4.1, we have that

$$
\bar{w} \in\left(\operatorname{Fix}\left(J_{\rho_{n}}^{G_{1}}\left(\left(I-\rho_{n} A_{1}^{*}\left(I-F_{1}\right) A_{1}\right)\right)\right) \cap \operatorname{Fix}\left(J_{\sigma_{n}}^{G_{2}}\left(\left(I-\sigma_{n} A_{2}^{*}\left(I-F_{2}\right) A_{2}\right)\right)\right)\right) \neq \emptyset
$$

for each $n \in \mathbb{N}$. This implies that

$$
\bar{w} \in\left(\operatorname{Fix}\left(J_{\rho_{n}}^{G_{1}}\left(I-\rho_{n} B_{1}\right)\right) \cap\left(\operatorname{Fix}\left(J_{\sigma_{n}}^{G_{2}}\left(I-\sigma_{n} B_{2}\right)\right)\right)\right) \neq \emptyset
$$

for each $n \in \mathbb{N}$. Hence,

$$
\bar{w} \in\left(\left(B_{1}+G_{1}\right)^{-1}(0) \cap\left(B_{2}+G_{2}\right)^{-1}(0)\right) \neq \emptyset .
$$

By Theorem 3.1, $\lim _{n \rightarrow \infty} v_{n}=\bar{x}$, where $\bar{x}=P_{\left(B_{1}+G_{1}\right)^{-1}(0) \cap\left(B_{2}+G_{2}\right)^{-1}(0)} u$. That is,

$$
\bar{x} \in\left(B_{1}+G_{1}\right)^{-1}(0) \cap\left(B_{2}+G_{2}\right)^{-1}(0)
$$

and

$$
\|\bar{x}-u\| \leq\|q-u\|
$$

for all $q \in\left(B_{1}+G_{1}\right)^{-1}(0) \cap\left(B_{2}+G_{2}\right)^{-1}(0)$. Since

$$
\bar{x} \in\left(B_{1}+G_{1}\right)^{-1}(0) \cap\left(B_{2}+G_{2}\right)^{-1}(0),
$$

we know that

$$
\bar{x} \in \operatorname{Fix}\left(J_{\rho_{n}}^{G_{1}}\left(I-\rho_{n} B_{1}\right)\right) \cap \operatorname{Fix}\left(J_{\sigma_{n}}^{G_{2}}\left(I-\sigma_{n} B_{2}\right)\right) .
$$

That is,

$$
\bar{x} \in \operatorname{Fix}\left(J_{\rho_{n}}^{G_{1}}\left(I-\rho_{n} A_{1}^{*}\left(I-F_{1}\right) A_{1}\right)\right)
$$


and

$$
\bar{x} \in \operatorname{Fix}\left(J_{\sigma_{n}}^{G_{2}}\left(I-\sigma_{n} A_{2}^{*}\left(I-F_{2}\right) A_{2}\right)\right) .
$$

By Lemma 4.1, we get that $\bar{x} \in \Omega_{A 1}$. Similarly, if $q \in\left(B_{1}+G_{1}\right)^{-1}(0) \cap\left(B_{2}+G_{2}\right)^{-1}(0)$, then $q \in \Omega_{A 1}$. Therefore $\bar{x}=P_{\Omega_{A 1}} u$. This shows that $\lim _{n \rightarrow \infty} v_{n}$ is a unique solution of the optimization problem

$$
\min _{q \in \Omega_{A 1}}\|q-u\|
$$

Therefore, the proof is completed.

In the following theorem, we study the following multiple sets split feasibility problem (MSSMVIP-A2):

Find $\bar{x} \in \mathcal{H}_{1}$ such that $\bar{x} \in C, A_{1} \bar{x} \in Q$ and $A_{2} \bar{x} \in Q^{\prime}$.

Let $\Omega_{A 2}$ denote the solution set of (MSSMVIP-A2). The following theorem is a special case of Theorem 4.3. Hence, it is also a special case of Theorem 4.2.

Theorem 4.3 Suppose that $\Omega_{A 2}$ is nonempty, and that $\left\{a_{n}\right\},\left\{b_{n}\right\},\left\{c_{n}\right\},\left\{f_{n}\right\},\left\{g_{n}\right\},\left\{h_{n}\right\}$ are sequences in $[0,1]$ with $a_{n}+b_{n}+c_{n}=1, f_{n}+g_{n}+h_{n}=1,0<a_{n}<1$, and $0<f_{n}<1$ for each $n \in \mathbb{N}$. For an arbitrary fixed $u \in \mathcal{H}$, a sequence $\left\{v_{n}\right\}$ is defined by

$$
\left\{\begin{array}{l}
v_{0} \in \mathcal{H} \text { is chosen arbitrarily, } \\
v_{2 n+1}:=a_{n} u+b_{n} v_{2 n}+c_{n} P_{C}\left(I-\rho_{n} A_{1}^{*}\left(I-P_{Q}\right) A_{1}\right) v_{2 n}, \quad n \in \mathbb{N} \cup\{0\} \\
v_{2 n}:=f_{n} u+g_{n} v_{2 n-1}+h_{n} P_{C}\left(I-\sigma_{n} A_{2}^{*}\left(I-P_{Q^{\prime}}\right) A_{2}\right) v_{2 n-1}, \quad n \in \mathbb{N}
\end{array}\right.
$$

Then $\lim _{n \rightarrow \infty} v_{n}$ is a unique solution of the following optimization problem:

$$
\min _{q \in \Omega_{A 2}}\|q-u\|
$$

provided the following conditions are satisfied:

(i) $\lim _{n \rightarrow \infty} a_{n}=\lim _{n \rightarrow \infty} f_{n}=0$;

(ii) either $\sum_{n=1}^{\infty} a_{n}=\infty$ or $\sum_{n=1}^{\infty} f_{n}=\infty$;

(iii) $0<a \leq \rho_{n} \leq b<\frac{2}{\left\|A_{1}\right\|^{2}+2}, 0<c \leq \sigma_{n} \leq d<\frac{2}{\left\|A_{2}\right\|^{2}+2}$ for each $n \in \mathbb{N}$ and for some $a, b, c, d \in \mathbb{R}^{+}$

(iv) $\liminf _{n \rightarrow \infty} c_{n}>0, \liminf _{n \rightarrow \infty} h_{n}>0$.

Proof Put $G_{1}=G_{2}=\partial \iota_{C}, F_{1}=P_{Q}$, and $F_{2}=P_{Q^{\prime}}$. Then $G_{1}, G_{2}$ are two set-valued maximum monotone mappings, $F_{1}$ and $F_{2}$ are firmly nonexpansive mappings. Since $J_{\rho_{n}}^{\partial \iota C}=P_{C}$ and $J_{\sigma_{n}}^{\partial \iota C}=P_{C}$, we have $\operatorname{Fix}\left(F_{1}\right)=\operatorname{Fix}\left(P_{Q}\right)=Q, \operatorname{Fix}\left(F_{2}\right)=\operatorname{Fix}\left(P_{Q^{\prime}}\right)=Q^{\prime}, \operatorname{Fix}\left(J_{\rho_{n}}^{\partial \iota^{C}}\right)=\operatorname{Fix}\left(P_{C}\right)=C$ and $\operatorname{Fix}\left(J_{\sigma_{n}}^{\partial \iota}\right)=\operatorname{Fix}\left(P_{C}\right)=C$. Then Theorem 4.3 follows from Theorem 4.2. 
In the following theorem, we study the following split feasibility problem (MSSMVIPA3):

Find $\bar{x} \in \mathcal{H}_{1}$ such that $\bar{x} \in C \cap Q^{\prime}, A_{1} \bar{x} \in Q$ where $Q^{\prime}$ is a nonempty closed subset of $\mathcal{H}_{1}$.

Let $\Omega_{A 3}$ denote the solution set of problem (MSSMVIP-A3). The following is also a special case of Theorem 4.3.

Theorem 4.4 Suppose that $Q^{\prime}$ is a nonempty closed convex subset of $\mathcal{H}_{1}, \Omega_{A 3}$ is nonempty, and $\left\{a_{n}\right\},\left\{b_{n}\right\},\left\{c_{n}\right\},\left\{f_{n}\right\},\left\{g_{n}\right\},\left\{h_{n}\right\}$ are sequences in $[0,1]$ with $a_{n}+b_{n}+c_{n}=1, f_{n}+g_{n}+h_{n}=1$, $0<a_{n}<1$, and $0<f_{n}<1$ for each $n \in \mathbb{N}$. For an arbitrary fixed $u \in \mathcal{H}$, a sequence $\left\{v_{n}\right\}$ is defined by

$$
\left\{\begin{array}{l}
v_{0} \in \mathcal{H} \text { is chosen arbitrarily, } \\
v_{2 n+1}:=a_{n} u+b_{n} v_{2 n}+c_{n} P_{C}\left(I-\rho_{n} A_{1}^{*}\left(I-P_{Q}\right) A_{1}\right) v_{2 n}, \quad n \in \mathbb{N} \cup\{0\} \\
v_{2 n}:=f_{n} u+g_{n} v_{2 n-1}+h_{n} P_{C}\left(I-\sigma_{n}\left(I-P_{Q^{\prime}}\right)\right) v_{2 n-1}, \quad n \in \mathbb{N}
\end{array}\right.
$$

Then $\lim _{n \rightarrow \infty} v_{n}$ is a unique solution of the following optimization problem:

$$
\min _{q \in \Omega_{A 3}}\|q-u\|
$$

provided the following conditions are satisfied:

(i) $\lim _{n \rightarrow \infty} a_{n}=\lim _{n \rightarrow \infty} f_{n}=0$;

(ii) either $\sum_{n=1}^{\infty} a_{n}=\infty$ or $\sum_{n=1}^{\infty} f_{n}=\infty$;

(iii) $0<a \leq \rho_{n} \leq b<\frac{2}{\left\|A_{1}\right\|^{2}+2}, 0<c<\sigma_{n}<d<\frac{2}{3}$ for each $n \in \mathbb{N}$ and for some $a, b, c, d \in \mathbb{R}^{+}$

(iv) $\liminf _{n \rightarrow \infty} c_{n}>0, \liminf _{n \rightarrow \infty} h_{n}>0$.

Proof Put $A_{2}=I$ and $\mathcal{H}_{1}=\mathcal{H}_{3}$ in Theorem 4.3. Then Theorem 4.4 follows from Theorem 4.3.

In the following theorem, we study the following convex feasibility problem (MSSMVIPA4):

Find $\bar{x} \in \mathcal{H}_{1}$ such that $\bar{x} \in C \cap Q \cap Q^{\prime}$, where $Q, Q^{\prime}$ are nonempty closed subsets of $\mathcal{H}_{1}$.

Let $\Omega_{A 4}$ denote the solution set of (MSSMVIP-A4). The following is a special case of Theorem 4.3.

Theorem 4.5 Suppose that $Q$ and $Q^{\prime}$ are nonempty closed convex subsets of $\mathcal{H}_{1}, \Omega_{A 4}$ is nonempty, and $\left\{a_{n}\right\},\left\{b_{n}\right\},\left\{c_{n}\right\},\left\{f_{n}\right\},\left\{g_{n}\right\},\left\{h_{n}\right\}$ are sequences in $[0,1]$ with $a_{n}+b_{n}+c_{n}=1$, $f_{n}+g_{n}+h_{n}=1,0<a_{n}<1$, and $0<f_{n}<1$ for each $n \in \mathbb{N}$. For an arbitrary fixed $u \in \mathcal{H}$, 
a sequence $\left\{v_{n}\right\}$ is defined by

$$
\left\{\begin{array}{l}
v_{0} \in \mathcal{H} \text { is chosen arbitrarily, } \\
v_{2 n+1}:=a_{n} u+b_{n} v_{2 n}+c_{n} P_{C}\left(I-\rho_{n}\left(I-P_{Q}\right)\right) v_{2 n}, \quad n \in \mathbb{N} \cup\{0\}, \\
v_{2 n}:=f_{n} u+g_{n} v_{2 n-1}+h_{n} P_{C}\left(I-\sigma_{n}\left(I-P_{Q^{\prime}}\right)\right) v_{2 n-1}, \quad n \in \mathbb{N} .
\end{array}\right.
$$

Then $\lim _{n \rightarrow \infty} v_{n}$ is a unique solution of the following optimization problem:

$$
\min _{q \in \Omega_{A 4}}\|q-u\|
$$

provided the following conditions are satisfied:

(i) $\lim _{n \rightarrow \infty} a_{n}=\lim _{n \rightarrow \infty} f_{n}=0$;

(ii) either $\sum_{n=1}^{\infty} a_{n}=\infty$ or $\sum_{n=1}^{\infty} f_{n}=\infty$;

(iii) $0<a<\rho_{n}<b<\frac{2}{3}, 0<c<\sigma_{n}<d<\frac{2}{3}$ for each $n \in \mathbb{N}$ and for some $a, b, c, d \in \mathbb{R}^{+}$;

(iv) $\liminf _{n \rightarrow \infty} c_{n}>0, \liminf _{n \rightarrow \infty} h_{n}>0$.

Proof Put $A_{1}=A_{2}=I$ and $\mathcal{H}_{1}=\mathcal{H}_{2}=\mathcal{H}_{3}$ in Theorem 4.3. Then Theorem 4.5 follows from Theorem 4.3.

In the following theorem, we study the following convex feasibility problem (MSSMVIPA5):

Find $\bar{x} \in \mathcal{H}_{1}$ such that $\bar{x} \in Q \cap Q^{\prime}$, where $Q$ and $Q^{\prime}$ are nonempty closed convex subsets of $\mathcal{H}_{1}$.

Let $\Omega_{A 5}$ denote the solution set of (MSSMVIP-A5).

The following existent theorem of a convex feasibility problem follows immediately from Theorem 4.5.

Theorem 4.6 Suppose that $Q$ and $Q^{\prime}$ are nonempty closed convex subsets of $\mathcal{H}_{1}, \Omega_{A 5}$ is nonempty, and $\left\{a_{n}\right\},\left\{b_{n}\right\},\left\{c_{n}\right\},\left\{f_{n}\right\},\left\{g_{n}\right\},\left\{h_{n}\right\}$ are sequences in $[0,1]$ with $a_{n}+b_{n}+c_{n}=1$, $f_{n}+g_{n}+h_{n}=1,0<a_{n}<1$, and $0<f_{n}<1$ for each $n \in \mathbb{N}$. For an arbitrary fixed $u \in \mathcal{H}$. Define a sequence $\left\{v_{n}\right\}$ by

$$
\left\{\begin{array}{l}
v_{0} \in \mathcal{H} \text { is chosen arbitrarily, } \\
v_{2 n+1}:=a_{n} u+b_{n} v_{2 n}+c_{n}\left(I-\rho_{n}\left(I-P_{Q}\right)\right) v_{2 n}, \quad n \in \mathbb{N} \cup\{0\} \\
v_{2 n}:=f_{n} u+g_{n} v_{2 n-1}+h_{n}\left(I-\sigma_{n}\left(I-P_{Q^{\prime}}\right)\right) v_{2 n-1}, \quad n \in \mathbb{N} .
\end{array}\right.
$$

Then $\lim _{n \rightarrow \infty} v_{n}$ is a unique solution of the following optimization problem:

$$
\min _{q \in \Omega_{A 5}}\|q-u\|
$$

provided the following conditions are satisfied:

(i) $\lim _{n \rightarrow \infty} a_{n}=\lim _{n \rightarrow \infty} f_{n}=0$;

(ii) either $\sum_{n=1}^{\infty} a_{n}=\infty$ or $\sum_{n=1}^{\infty} f_{n}=\infty$; 
(iii) $0<a<\rho_{n}<b<\frac{2}{3}, 0<c<\sigma_{n}<d<\frac{2}{3}$ for each $n \in \mathbb{N}$ and for some $a, b, c, d \in \mathbb{R}^{+}$;

(iv) $\liminf _{n \rightarrow \infty} c_{n}>0, \liminf _{n \rightarrow \infty} h_{n}>0$.

Proof Put $C=\mathcal{H}_{1}$, then $P_{C}=P_{\mathcal{H}_{1}}$. Then Theorem 4.6 follows from Theorem 4.5.

In the following theorem, we study the following system of convexly constrained linear inverse problem (SCCLIP):

Find $\bar{x} \in \mathcal{H}_{1}$ such that $\bar{x} \in C, A_{1} \bar{x}=b$ and $A_{2} \bar{x}=b^{\prime}$, where $b \in \mathcal{H}_{2}$ and $b^{\prime} \in \mathcal{H}_{3}$.

Let $\Omega_{A 6}$ denote the solution set of (SCCLIP).

Theorem 4.7 Suppose that $\Omega_{A 6}$ is nonempty, and $b \in \mathcal{H}_{2}, b^{\prime} \in \mathcal{H}_{3} . \operatorname{Let}\left\{a_{n}\right\},\left\{b_{n}\right\},\left\{c_{n}\right\},\left\{f_{n}\right\}$, $\left\{g_{n}\right\}$, and $\left\{h_{n}\right\}$ be sequences in $[0,1]$ with $a_{n}+b_{n}+c_{n}=1, f_{n}+g_{n}+h_{n}=1,0<a_{n}<1$, and $0<f_{n}<1$ for each $n \in \mathbb{N}$. For an arbitrary fixed $u \in \mathcal{H}$, a sequence $\left\{v_{n}\right\}$ is defined by

$$
\left\{\begin{array}{l}
v_{0} \in \mathcal{H} \text { is chosen arbitrarily, } \\
v_{2 n+1}:=a_{n} u+b_{n} v_{2 n}+c_{n} P_{C}\left(v_{2 n}-\rho_{n} A_{1}^{*}\left(A_{1} v_{2 n}-b\right)\right), \quad n \in \mathbb{N} \cup\{0\}, \\
v_{2 n}:=f_{n} u+g_{n} v_{2 n-1}+h_{n} P_{C}\left(v_{2 n-1}-\sigma_{n} A_{2}^{*}\left(A_{2} v_{2 n-1}-b^{\prime}\right)\right), \quad n \in \mathbb{N} .
\end{array}\right.
$$

Then $\lim _{n \rightarrow \infty} v_{n}$ is a unique solution of the following optimization problem:

$$
\min _{q \in \Omega_{A 6}}\|q-u\|
$$

provided the following conditions are satisfied:

(i) $\lim _{n \rightarrow \infty} a_{n}=\lim _{n \rightarrow \infty} f_{n}=0$;

(ii) either $\sum_{n=1}^{\infty} a_{n}=\infty$ or $\sum_{n=1}^{\infty} f_{n}=\infty$;

(iii) $0<a \leq \rho_{n} \leq b<\frac{2}{\left\|A_{1}\right\|^{2}+2}, 0<c \leq \sigma_{n} \leq d<\frac{2}{\left\|A_{2}\right\|^{2}+2}$ for each $n \in \mathbb{N}$ and for some $a, b, c, d \in \mathbb{R}^{+}$

(iv) $\liminf _{n \rightarrow \infty} c_{n}>0, \liminf _{n \rightarrow \infty} h_{n}>0$.

Proof Put $Q=\{b\}$ and $Q^{\prime}=\left\{b^{\prime}\right\}$. Then Theorem 4.7 follows from Theorem 4.2.

In the following theorem, we study the following convexly constrained linear inverse problem (CCLIP):

Find $\bar{x} \in \mathcal{H}_{1}$ such that $\bar{x} \in C \cap Q^{\prime}$ and $A_{1} \bar{x}=b$, where $b \in \mathcal{H}_{2}$ and $Q^{\prime}$ is a nonempty closed convex subset of $\mathcal{H}_{1}$.

Let $\Omega_{A 7}$ denote the solution set of (CCLIP).

Theorem 4.8 Suppose that $Q^{\prime}$ is a nonempty closed convex subset of $\mathcal{H}_{1} . \Omega_{A 7}$ is nonempty, $b \in \mathcal{H}_{2}$, and $\left\{a_{n}\right\},\left\{b_{n}\right\},\left\{c_{n}\right\},\left\{f_{n}\right\},\left\{g_{n}\right\},\left\{h_{n}\right\}$ are sequences in $[0,1]$ with $a_{n}+b_{n}+c_{n}=1$, $f_{n}+g_{n}+h_{n}=1,0<a_{n}<1$, and $0<f_{n}<1$ for each $n \in \mathbb{N}$. For an arbitrary fixed $u \in \mathcal{H}$, a sequence $\left\{v_{n}\right\}$ is defined by

$$
\begin{cases}v_{0} \in \mathcal{H} \text { is chosen arbitrarily, } \\ v_{2 n+1}:=a_{n} u+b_{n} v_{2 n}+c_{n} P_{C}\left(v_{2 n}-\rho_{n}\left(A_{1} v_{2 n}-b\right)\right), & n \in \mathbb{N} \cup\{0\}, \\ v_{2 n}:=f_{n} u+g_{n} v_{2 n-1}+h_{n} P_{C}\left(I-\sigma_{n}\left(I-P_{Q^{\prime}}\right)\right) v_{2 n-1}, & n \in \mathbb{N}\end{cases}
$$


Then $\lim _{n \rightarrow \infty} v_{n}$ is a unique solution of the following optimization problem:

$$
\min _{q \in \Omega_{A 7}}\|q-u\|
$$

provided the following conditions are satisfied:

(i) $\lim _{n \rightarrow \infty} a_{n}=\lim _{n \rightarrow \infty} f_{n}=0$;

(ii) either $\sum_{n=1}^{\infty} a_{n}=\infty$ or $\sum_{n=1}^{\infty} f_{n}=\infty$;

(iii) $0<a \leq \rho_{n} \leq b<\frac{2}{\left\|A_{1}\right\|^{2}+2}, 0<c<\sigma_{n}<d<\frac{2}{3}$ for each $n \in \mathbb{N}$, and for some

$$
a, b, c, d \in \mathbb{R}^{+}
$$

(iv) $\liminf _{n \rightarrow \infty} c_{n}>0, \liminf _{n \rightarrow \infty} h_{n}>0$.

Remark 4.2 The iteration in Theorem 4.8 is different from the Landweber iterative method [19]:

$$
x_{n+1}:=x_{n}+\gamma A^{T}\left(b-A x_{n}\right), \quad n \in \mathbb{N} .
$$

\section{Competing interests}

The authors declare that they have no competing interests.

\section{Authors' contributions}

L-JL designed and coordinated this research project and revised the paper. Y-DC carried out the project, drafted and revised the manuscript. C-SC coordinated the project and revised the paper.

\section{Author details}

'Department of Mathematics, National Changhua University of Education, Changhua, 50058, Taiwan. ${ }^{2}$ Department of Chemical and Materials Engineering, Southern Taiwan University of Science and Technology, Tainan, 71005, Taiwan.

${ }^{3}$ Department of Applied Mathematics, National Sun Yat-sen University, Kaohsiung, Taiwan.

\section{Acknowledgements}

Prof. C-S Chuang was supported by the National Science Council of Republic of China while he work on the publish, and Y-D Chen was supported by Southern Taiwan University of Science and Technology.

Received: 3 April 2013 Accepted: 7 November 2013 Published: 06 Dec 2013

\section{References}

1. Combettes, PL: The convex feasible problem in image recovery. In: Hawkes, P (ed.) Advanced in Image and Electron Physics, vol. 95, pp. 155-270. Academic Press, New York (1996)

2. Stark, H: Image Recovery: Theory and Applications. Academic Press, New York (1987)

3. Bregman, LM: The relaxation method of finding the common point of convex sets and its application to the solution of problems in convex programming. USSR Comput. Math. Math. Phys. 7, 200-217 (1967)

4. Hundal, $\mathrm{H}$ : An alternating projection that does not converge in norm. Nonlinear Anal. 57, 35-61 (2004)

5. Boikanyo, OA, Morosanu, G: Strong convergence of the method of alternating resolvents. J. Nonlinear Convex Anal. $14,221-229(2013)$

6. Censor, Y, Elfving, T: A multiprojection algorithm using Bregman projection in a product space. Numer. Algorithms 8 , 221-239 (1994)

7. Bauschke, HH, Borwein, JM: On projection algorithm for solving convex feasible problems. SIAM Rev. 38, 376-426 (1996)

8. Byrne, C: Iterative oblique projection onto convex sets and the split feasibility problem. Inverse Probl. 18, 441-453 (2002)

9. Byrne, C: A unified treatment of some iterative algorithms in signal processing and image reconstruction. Inverse Probl. 20, 103-120 (2004)

10. Censor, Y, Bortfeld, T, Martin, B, Trofimov, A: A unified approach for inversion problems in intensity modulated radiation therapy. Phys. Med. Biol. 51, 2353-2365 (2003)

11. Censor, Y, Elfving, T, Kopf, N, Bortfeld, T: The multiple-sets split feasibility problem and its applications for inverse problems. Inverse Probl. 21, 2071-2084 (2005)

12. López, G, Martín-Márquez, V, Xu, HK: Iterative algorithms for the multiple-sets split feasibility problem. In: Censor, Y, Jiang, M, Wang, G (eds.) Biomedical Mathematics: Promising Directions in Imaging, Therapy Planning and Inverse Problems, pp. 243-279. Medical Physics Publishing, Madison (2010)

13. Lopez, G, Martinez-Marquez, V, Wang, F, Xu, HK: Solving the split feasibility problem without prior knowledge of matrix n. Inverse Probl. 28, Article ID 085004 (2012)

14. Qu, B, Xiu, N: A note on the CQ algorithm for the split feasibility problem. Inverse Probl. 21, 1655-1665 (2005) 
15. Xu, HK: Iterative methods for the split feasibility problem in infinite-dimensional Hilbert spaces. Inverse Probl. 26, Article ID 105018 (2010)

16. Xu, HK: Iterative methods for the split feasibility problem in infinite-dimensional Hilbert spaces. Inverse Probl. 26, Article ID 105018 (2010)

17. Yang, Q: The relaxed CQ algorithm solving the split feasible problem. Inverse Probl. 20, 1261-1266 (2004)

18. Eicke, B: Iteration methods for convexly constrained ill-posed problems in Hilbert space. Numer. Funct. Anal. Optim. $13,413-429(1992)$

19. Landweber, L: An iteration formula for Fredholm integral equations of the first kind. Am. J. Math. 73, 615-624 (1951)

20. Boikanyo, OA, Morosanu, G: A contraction proximal point algorithm with two monotone operators. Nonlinear Anal. 75, 5686-5692 (2012)

21. Kamimura, S, Takahashi, W: Approximating solutions of maximal monotone operators in Hilbert spaces. J. Approx. Theory 106, 226-240 (2000)

22. Xu, HK: Iterative algorithm for nonlinear operators. J. Lond. Math. Soc. 2, 240-256 (2002)

23. Yao, Y, Noor, MA: On convergence criteria of generalized proximal point algorithms. J. Comput. Appl. Math. 217, 46-55 (2008)

24. Wang, F, Cui, H: On the contraction-proximal point algorithms with multi-parameters. J. Glob. Optim. 54, 485-491 (2012)

25. Yu, ZT, Lin, LJ, Chuang, CS: A Unified Study of The Split Feasible Problems With Applications. J. Nonlinear Convex Anal. (to appear)

26. Takahashi, W: Nonlinear Functional Analysis-Fixed Point Theory and Its Applications. Yokohama Publishers, Yokohama (2000)

27. Marino, G, Xu, HK: Convergence of generalized proximal point algorithm. Commun. Pure Appl. Anal. 3, 791-808 (2004)

28. Maingé, PE: Strong convergence of projected subgradient methods for nonsmooth and nonstrictly convex minimization. Set-Valued Anal. 16, 899-912 (2008)

29. Combettes, PL: Solving monotone inclusions via compositions of nonexpansive averaged operators. Optimization 53, 475-504 (2004)

30. Goebel, A, Kirk, WA: Topics in Metric Fixed Point Theory. Cambridge University Press, Cambridge (1990)

31. Rockafellar, RT: Monotone operators and the proximal point algorithm. SIAM J. Control Optim. 14, 877-898 (1976)

32. Boikanyo, OA, Morosanu, G: Inexact Halpern-type proximal point algorithms. J. Glob. Optim. 57, 11-26 (2011)

33. Yu, ZT, Lin, LZ: Hierarchical problem with applications to mathematical programming with multiple sets split feasibility constraints. Fixed Point Theory Appl. 2013, Article ID 283 (2013). doi:10.1186/1687-1812-2013-283

10.1186/1687-1812-2013-333

Cite this article as: Lin et al.: Solutions for a variational inclusion problem with applications to multiple sets split feasibility problems. Fixed Point Theory and Applications 2013, 2013:333

\section{Submit your manuscript to a SpringerOpen ${ }^{\ominus}$ journal and benefit from:}

- Convenient online submission

Rigorous peer review

- Immediate publication on acceptance

- Open access: articles freely available online

- High visibility within the field

- Retaining the copyright to your article 